\title{
Crystal structures and phase transitions of long-chain $n$-alkylammonium bromide monohydrates $\dagger$
}

\author{
Melanie Rademeyer, ${ }^{* a}$ Gert J. Kruger ${ }^{b}$ and David G. Billing ${ }^{c}$ \\ Received 3rd October 2008, Accepted 8th May 2009 \\ First published as an Advance Article on the web 27th May 2009 \\ DOI: 10.1039/b817318d
}

The crystal structures of five long-chain $n$-alkylammonium bromide monohydrates, with carbon chain lengths ranging from 13 to 18 , were determined. $n$-Tridecylammonium bromide monohydrate $\mathbf{1}$,

$n$-tetradecylammonium bromide monohydrate $\mathbf{2}, n$-pentadecylammonium bromide monohydrate $\mathbf{3}$, $n$-hexadecylammonium bromide monohydrate $\mathbf{4}$, and $n$-octadecylammonium bromide monohydrate $\mathbf{5}$, all crystallise in layered structures, and the same hydrogen bonding network is observed for the oddand even carbon chain compounds. Comparison of an anhydrous and a hydrated $n$-alkylammonium bromide structure indicate that the structures differ in alkyl chain tilt angle, methylene subcell packing as well as hydrogen bonding network. The thermotropic phase transitions exhibited by the hydrates were investigated via DSC and TGA, and indicate stepwise melting behaviour through a number of high temperature phases, including a liquid crystalline phase.

\section{Introduction}

Long-chain molecules, containing a substantial alkyl section, constitute many commercially important substances including detergents, fatty acids and waxes. Typically these compounds selfassemble in layers with the alkyl parts packing in parallel to maximise lateral hydrophobic interactions. The crystal structures of a number of terminally monosubstituted long-chain systems ${ }^{1}$ and terminally di-substituted long-chain families ${ }^{2}$ have been reported.

Another interesting feature of long-chain compounds is the occurrence of stepwise melting behaviour, including thermotropic polymorphism. Solid-solid phase transitions to more disordered solids and/or liquid crystal phases often take place before complete melting occurs. ${ }^{3}$

The observation of alternating phase transition temperatures, melting points and packing densities of odd-carbon members in a long-chain series compared to the even-carbon members is called the "odd-even effect", and has been shown to exist for many long-chain systems. ${ }^{4}$ A geometrical, two-dimensional parallelogram and trapezoid model, proposed by Boese, ${ }^{5}$ where odd carbon chain molecules (containing 2-fold rotational symmetry) are approximated by trapezoids, and centrosymmetric even carbon chain molecules by parallelograms, highlighted the origin of this effect for a number of long-chain families. For the $n$-alkanes, ${ }^{5}$ the $\alpha, \omega$-alkanediols, ${ }^{6}$ the $\alpha, \omega$-alkanediamines, ${ }^{6}$ the $\alpha, \omega$-alkanedithiols ${ }^{7}$ and the $n$-alkyl carboxylic $\operatorname{acids}^{8}$ it was shown that unfavourable packing and off-set of the

${ }^{a}$ Department of Chemistry, University of Pretoria, Pretoria, 0002, South Africa. E-mail: melanie.rademeyer@up.ac.za; Fax: +2712 420 4687; Tel: +27124202511

${ }^{b}$ Department of Chemistry, University of Johannesburg, P.O. Box 524, Auckland Park, 2006, South Africa. E-mail: gjkruger@uj.ac.za; Fax: + 2711559 2819; Tel: + 27115592368

'Molecular Science Institute, School of Chemistry, University of the Witwatersrand, Private Bag 3, PO Wits, 2050, South Africa. E-mail: Dave.Billing@wits.ac.za; Fax: +2711 717 6749; Tel: +2711 7176749 $\dagger$ CCDC reference numbers 704568-704572. For crystallographic data in CIF or other electronic format see DOI: $10.1039 / \mathrm{b} 817318 \mathrm{~d}$ terminal parts of the molecules on the layer surface occurred for the odd members, resulting in lower melting points and densities for these compounds. In the $\alpha, \omega$-alkanedicarboxylic acid series ${ }^{9}$ the melting points of odd members are lower than those of the even members, but their densities are higher, contrary to what is observed in other systems. This observation was not ascribed to close packing, but to energetically unfavourable molecular conformations being present in the odd member structures.

Primary long-chain n-alkylammonium halides $\left(\mathrm{CH}_{3}\left(\mathrm{CH}_{2}\right)_{\mathrm{n}}\right.$ $\mathrm{NH}_{3}{ }^{+} \mathrm{X}^{-} \cdot \mathrm{pH}_{2} \mathrm{O}$, where $\mathrm{n}$ exceeds nine, $\mathrm{X}$ is a halide anion, and $\mathrm{p}$ is zero or one) will self-assemble in the solid state into hydrophobic and hydrophilic layers. Cohesion occurs via hydrophobic interactions between alkyl moeities in the hydrophobic layers and through charge assisted hydrogen bonding in the hydrophilic layers.

The single crystal structures of three anhydrous, long-chain $n$-alkylammonium halides have been reported in the literature, namely $n$-decylammonium chloride, ${ }^{10} n$-dodecylammonium chloride $^{11}$ and $n$-dodecylammonium bromide. ${ }^{12}$

Two long-chain $n$-alkylammonium halide hydrate structures have been reported, namely $n$-undecylammonium chloride monohydrate ${ }^{13}$ and $n$-undecylammonium bromide monohydrate. ${ }^{14}$ However, no hydrate or anhydrous structure of an $n$-alkylammonium halide with more than twelve carbon atoms has to date been reported.

The long-chain $n$-alkylammonium halides exhibit a number of thermally induced solid-solid phase transitions to high temperature phases due to stepwise melting behaviour. The thermotropic phase transitions of the long-chain $n$-alkylammonium chlorides, particularly those of $n$-decylammonium chloride, have been investigated extensively. ${ }^{15}$ Five solid phases have been observed between $-195{ }^{\circ} \mathrm{C}$ and $260{ }^{\circ} \mathrm{C}$ for $n$-decylammonium chloride, and characterised to various degrees. The solutioncrystallized room temperature form, called the $i$ form, is interdigitated and ordered. ${ }^{8}$ An irreversible transition to a phase called the $\delta$ phase involves a change to a layered, non-interdigitated structure with conformational defects present in the chains. 
On further heating a transition to the $\beta$ phase results in a layered structure with alkyl chains perpendicular to the hydrophilic layer, and even more disordered alkyl chains, followed by a transition to the $\alpha$ phase that displays a large amount of disorder in the alkyl chains. The $\alpha$ phase undergoes a transition to a liquid crystal phase, followed by melting. Cooling of the melted material to room temperature yields the $\epsilon$ phase, characterized by the presence of conformational defects in the chains. Cooling of the $\epsilon$ phase below room temperature causes a denser packing and more ordered hydrocarbon chains.

In contrast to the $n$-alkylammonium chlorides, only one thermal analysis study,${ }^{16}$ carried out in 1968 , has focussed on the $n$-alkylammonium bromides with carbon chain lengths ranging from 1 to 16 .

The aim of the present study was to structurally characterize $n$ alkylammonium bromides with carbon chain lengths exceeding twelve carbon atoms (compounds $\mathbf{1}$ to $\mathbf{5}$, Table 1), and to investigate the tendency of these compounds to incorporate water molecules into their hydrophilic layers, and the effect this has on the hydrogen bonding network. In addition, the thermotropic phase transitions exhibited specifically by the hydrated forms were investigated to obtain information on the existence of high temperature phases of the longer chain $n$-alkylammonium bromides.

\section{Results and discussion}

\section{Description of structures 1 to 5}

All the $n$-alkylammonium bromide crystals studied in this investigation had water molecules incorporated in their hydrophilic layers. Infantes et $a l^{17}$ calculated a "water affinity" parameter that indicates the frequency with which hydrates are encountered in combination with specific species or functional groups. It was shown that this parameter has a value of around $30 \%$ for the ammonium group, and approximately $22 \%$ for the bromide anion. No comment was made regarding the water affinity of the combination of a bromide anion and an ammonium group. The incorporation of water molecules is most probably due to the improved packing achieved by the inclusion of water molecules with positional freedom in the hydrogen bonding network.

The asymmetric units of compounds $\mathbf{1}$ to $\mathbf{5}$ all contain one $n$-alkylammonium cation, a bromide anion and one water molecule, and are illustrated in Fig 1. Crystallographic data for structures $\mathbf{1}$ to $\mathbf{5}$ are listed in Table 1 .

Compounds $\mathbf{1}$ to $\mathbf{5}$ self-assemble to form layered structures, with the hydrophilic layer containing the water molecules, ammonium groups and bromide anions. The alkyl moeity of the $n$-alkylammonium cation comprise the interdigitated hydrophobic layer.

The structure obtained is a compromise between two main conflicting intermolecular interactions. The long chains aim to pack in parallel to maximise lateral hydrophobic interactions, while the headgroups in the hydrophilic layer aim to be positioned such as to achieve optimum hydrogen bonding interactions. The importance of the hydrophobic interactions relative to the hydrogen bonding interactions is expected to increase when the chain length increases. The observed structure is the result of

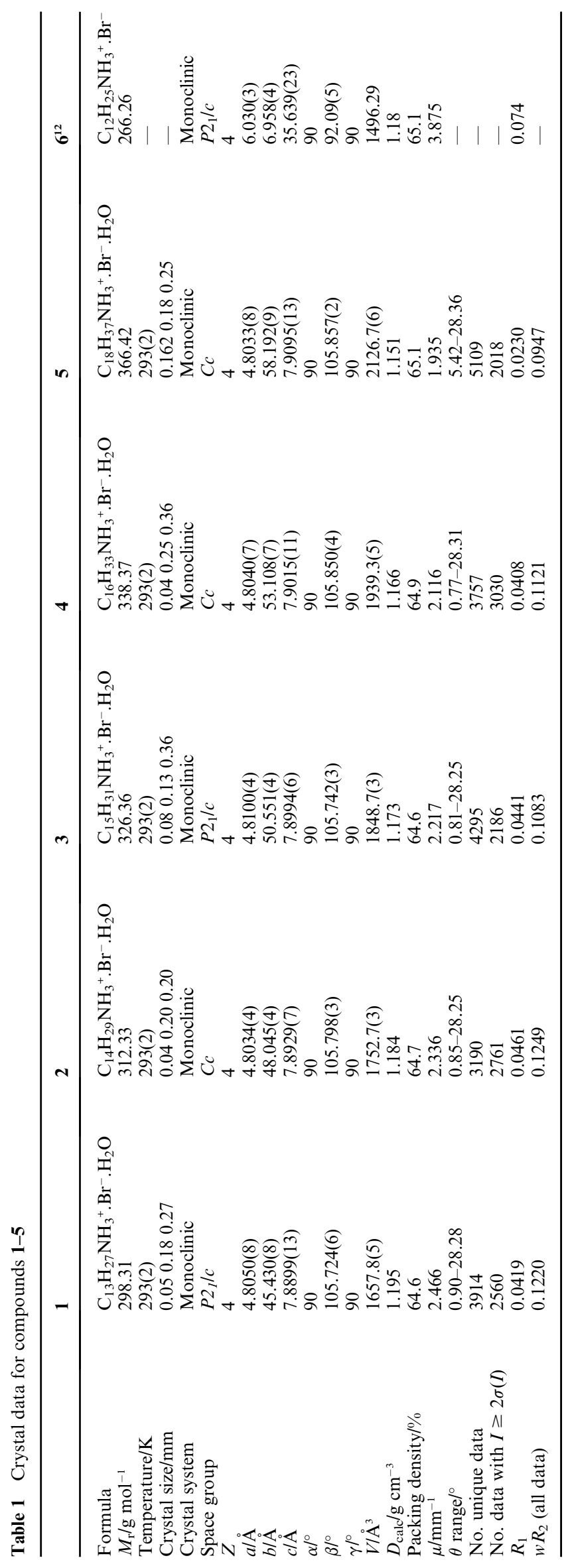




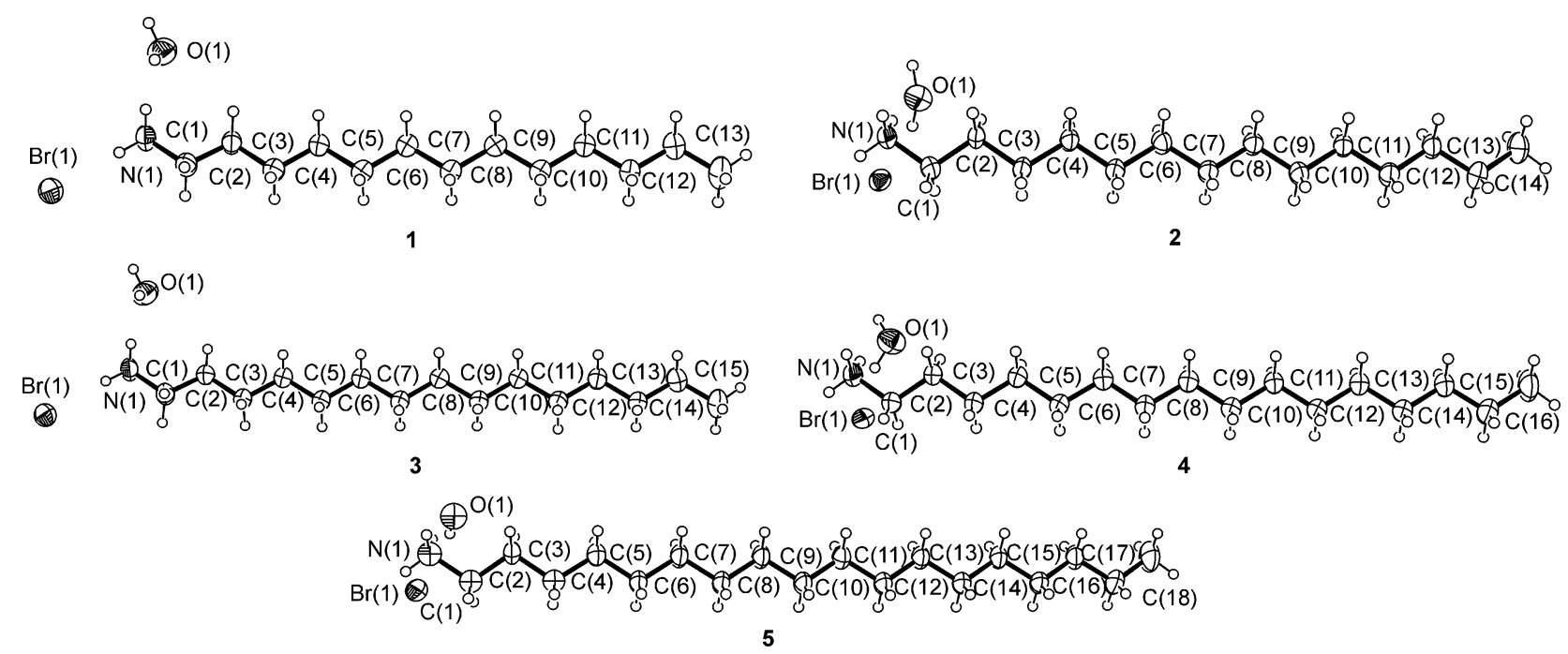

Fig. 1 Asymmetric units of compounds 1 to 5 showing the atomic numbering scheme. Thermal ellipsoids are illustrated at the $50 \%$ probability level.

a delicate balance between these two interactions, which is evident from the molecular geometries observed.

In all the structures the long aliphatic chain is in the low energy, extended, all-trans conformation, with most of the bond angles and torsion angles in the alkyl chain being close to the ideal values of $112^{\circ}$ and $180^{\circ}$ respectively. In the region of the ammonium group, however, the flexible chains curve out from the mean plane defined by the carbon atoms, orienting the ammonium headgroup in such a way as to create the optimal hydrogen bonding network. Thus, while the hydrocarbon chains are, on average, perpendicular to the layer plane, the $\mathrm{N}(1)-\mathrm{C}(1)-$ $\mathrm{C}(2)$ vector is at an angle to it. The observed molecular geometry is a compromise between maximising hydrophobic and hydrogen bonding interactions. Table 2 lists the angle the $\mathrm{N}(1)-\mathrm{C}(1)-\mathrm{C}(2)$ vector makes with the layer plane, the deviations of atoms from the carbon zig-zag plane and the deviation of torsion angles from their ideal values in the region of the ammonium group. Atoms of the even carbon chain compounds exhibit a larger deviation from the $\mathrm{C}-\mathrm{C}$ plane than odd carbon chain compounds, which is also expressed as a slightly larger $\mathrm{N}(1)-\mathrm{C}(1)-\mathrm{C}(2)$ vector to layerplane angle. The magnitude of the deviation from the all-trans conformation increases with an increase in chain length because the hydrophobic interactions become more important relative to hydrogen bonding in the longer chain compounds. A similar effect was observed in the $n$-alkylcarboxylic acid family. ${ }^{8}$

The degree of interdigitation is the same in all the structures, i.e. chain overlap commencing at atom $\mathrm{C}(3)$. The hydrocarbon chain packing in the all-trans, interdigitated regions can be described by the $\mathrm{M}_{2}{ }^{\|}$methylene subcell ${ }^{18}$ for all the compounds (average subcell parameters: $a_{s}=4.80 \AA b_{s}=8.050 \AA$ and $\gamma_{s}=109.28 \AA$ ), with the carbon zig-zag planes parallel to maximise the van der Waals interactions, and an average crosssectional area per chain of $18.25 \AA^{2}$. This means that the packing density in the hydrophobic region of the structure is similar for all the compounds.

The three even carbon chain compounds $\mathbf{2 , 4}$ and $\mathbf{5}$ crystallize in the space group $C c$ while the odd carbon chain hydrates $\mathbf{1}$ and $\mathbf{3}$ crystallize in space group $P 2_{1} / c$. For all five structures the $a$ and $c$ unit cell parameters are approximately equal, whilst the $b$ unit cell parameter increases linearly to accommodate the increase in alkyl chain length, with an average increase of approximately $2.6 \AA$ per alkyl carbon $(b(\AA)=2.58 n+12.33$, where $n$ is the number of carbon atoms). There is a linear decrease in density of the structures as the alkyl chain length increases (Table 1) reflecting the increased relative contribution of the longer alkyl chain.

The layered structure of $\mathbf{1}$ and $\mathbf{2}$, representatives of the odd and even carbon chain structures, are shown in Fig. $2 \mathrm{a}$ and c. In the even chain structures (space group $C c$ ) the ammonium headgroups point in the same direction resulting in a $c$-glide plane perpendicular to the $b$-axis, in the centre of the hydrophobic layer (Fig. 2b). In the odd carbon chain structures (space group $P 2_{1} / c$ ), shown in Fig. 2d, an inversion centre in the middle of the hydrophobic layer results from the headgroups pointing in opposite directions.

The source of the different space groups for even- and odd chain members is the requirement for alkyl chain interdigitation to start at atom C(3), exhibited by all compounds, regardless of space group. As illustrated in Fig. 2f, when an odd carbon chain interdigitates with another odd-carbon chain a centrosymmetric pair results, thus requiring a centre of inversion in the space group. However, when two even carbon chains interdigitate in the same manner, the pair is non-centrosymmetric, as illustrated in Fig. 2g. Thus, the molecular symmetry and interdigitation requirements are extended to the overall symmetry of the structures.

Exactly the same orientation is adopted by terminal methyl groups and hydrogen bonding moeities at the layer interface in the even- and odd carbon structures. This results in the same hydrogen bonding network forming around the $c$-glide planes perpendicular to the $b$-axis present in all compounds, i.e. in both $C c$ and $P 2_{1} / c$. This observation is contrary to what is observed in many long-chain systems ${ }^{5-9}$ where the odd- and even carbon compounds differ in the packing of the terminal groups at the layer interface.

It was observed that the following similarities between oddand even chain compounds exist: (a) the same average molecular 
geometry (b) the same interlayer packing and hydrogen bonding network (c) no alternation in density (e) the same degree of interdigitation (f) a linear increase in $b$ unit cell parameter. These results indicate that, rather than viewing the current set of structures as consisting of two different homologous series, one containing the odd carbon members and the other containing the even carbon members, they may be viewed to be a pseudocontinuous series of structures with small packing differences. The reason that the odd-even effect is not a prominent feature of the structures may be attributed to the fact that the presence of isolated water molecules and bromide anions allow for more flexibility in the structures, specifically on the interlayer surface, in contrast to the neutral molecules fitting the parallelogramtrapezoid model.

Strong charge assisted $\mathrm{N}^{+}-\mathrm{H} \cdots \mathrm{Br}^{-}, \mathrm{N}^{+}-\mathrm{H} \cdots \mathrm{O}$ and $\mathrm{O}-\mathrm{H} \cdots$ $\mathrm{Br}^{-}$hydrogen bonds are present in the hydrophilic layers, with hydrogen bonding parameters listed in Table 3.

The ammonium group and water molecule both act as threeconnecting nodes, with the ammonium group hydrogen bonding to two bromide anions and one water molecule, and the water molecule hydrogen bonding to two bromide anions and one ammonium group. The bromide anion functions as a four connecting node, connecting two water molecules and two ammonium groups.

The concept of ring-laddering may be employed to describe the resulting hydrogen bonding network. ${ }^{19}$ A distorted hydrogen bonded ladder can be defined as follows: $\mathrm{N}^{+}-\mathrm{H} \cdots \mathrm{Br}^{-}$and $\mathrm{N}^{+}-\mathrm{H} \cdots \mathrm{O}$ interactions form the ladder arms, and $\mathrm{O}-\mathrm{H} \cdots \mathrm{Br}^{-}$ interactions the ladder rungs, as illustrated in Fig. 3a. The resulting ring motif can be described by level four graph set notation as $\mathrm{R}_{8}^{5}(16) .{ }^{20}$ Neighbouring ladders are linked by $\mathrm{O}-\mathrm{H} \cdots$ $\mathrm{Br}^{-}$interactions to form a corrugated, two-dimensional cis net, with a cation on the same side of the net as its neighbours (Fig. 3b). Two such infinite nets are further associated by $\mathrm{N}-\mathrm{H}^{+} \cdots \mathrm{Br}^{-}$interactions to form a ionic, hydrophilic bilayer, illustrated in Fig. 3c.

\section{Comparison of hydrate and anhydrous structures}

The effect of the incorporation of water molecules into the longchain $n$-alkylammonium bromide structures may be studied by comparing the crystal structure of the previously-published anhydrous $n$-dodecylammonium bromide, ${ }^{12}$ compound $\mathbf{6}$, with the closest hydrate, the even carbon chain hydrated structure, 2. The most prominent difference between $\mathbf{2}$ and $\mathbf{6}$ is the tilt angle of the interdigitated alkyl chains relative to the layer plane (Fig. 2c and e). In 2 the chains are almost perpendicular to the hydrophilic plane (tilt angle: $89.8^{\circ}$ ) whereas in 6 , the chains are tilted by $52.1^{\circ}$.

Another major difference pertains to the hydrocarbon chain packing. ${ }^{9}$ In 2 the $\mathbf{M}^{\|}$methylene subcell is exhibited, with all hydrocarbon axes and carbon zigzag planes parallel, and a crosssectional area per alkyl chain of $18.24 \AA^{2}$, as shown in Fig. 4a. The alkyl chains in anhydrous structure 6 adopt the $\mathrm{O} \perp^{\prime}$ methylene subcell, where the chain axes are parallel, but with perpendicular carbon zigzag planes, and a cross-sectional area of $18.80 \AA^{2}$ (illustrated in Fig. 4b). The cross-sectional area per ammonium group and bromide anion of $41.9 \AA^{2}$ in 6 exceeds the $36.5 \AA^{2}$ occupied by the ammonium group, water molecule and 


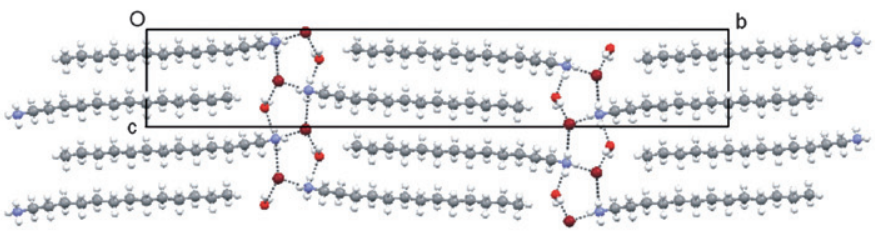

(a)

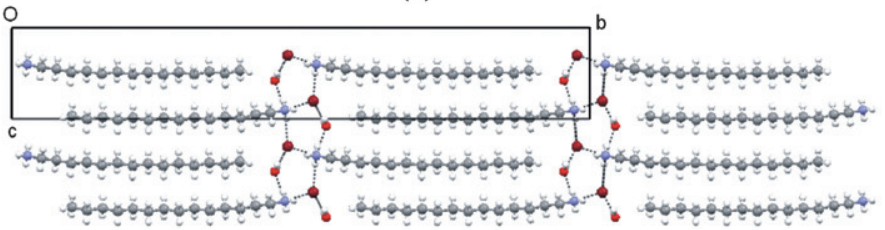

(c)

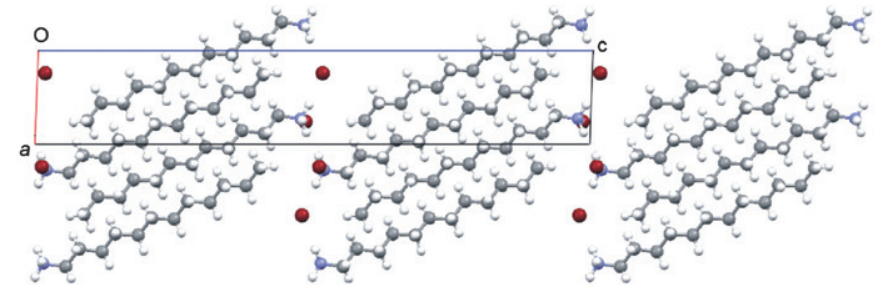

(e)

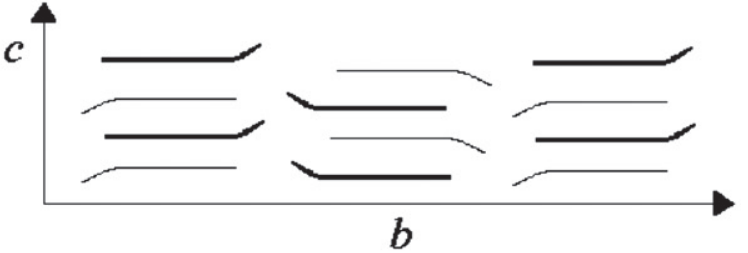

(b)

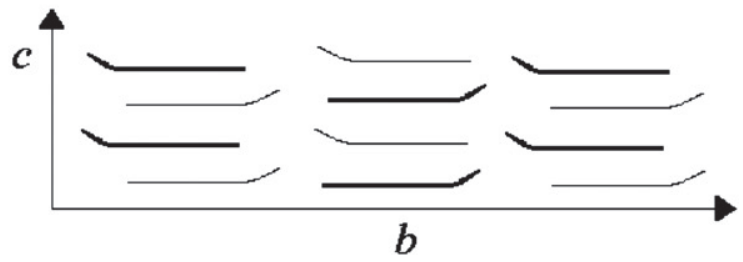

(d)

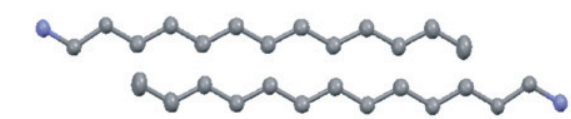

(f)

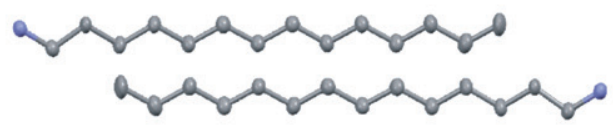

(g)

Fig. 2 (a) Layered packing of $\mathbf{1}$ and (b) schematic representation of packing in odd carbon chain structures (c) layered packing in $\mathbf{2}$, and (d) schematic representation of packing in even carbon chain structures. (e) Layered packing showing tilted chains in structure 6. (f) Centrosymmetric interdigitated odd carbon chain pair in $\mathbf{1}$ and $(\mathrm{g})$ non-centrosymmetric interdigitated even carbon chain pair in $\mathbf{2}$.

Table 3 Hydrogen bonding parameters for structures 1 to 5

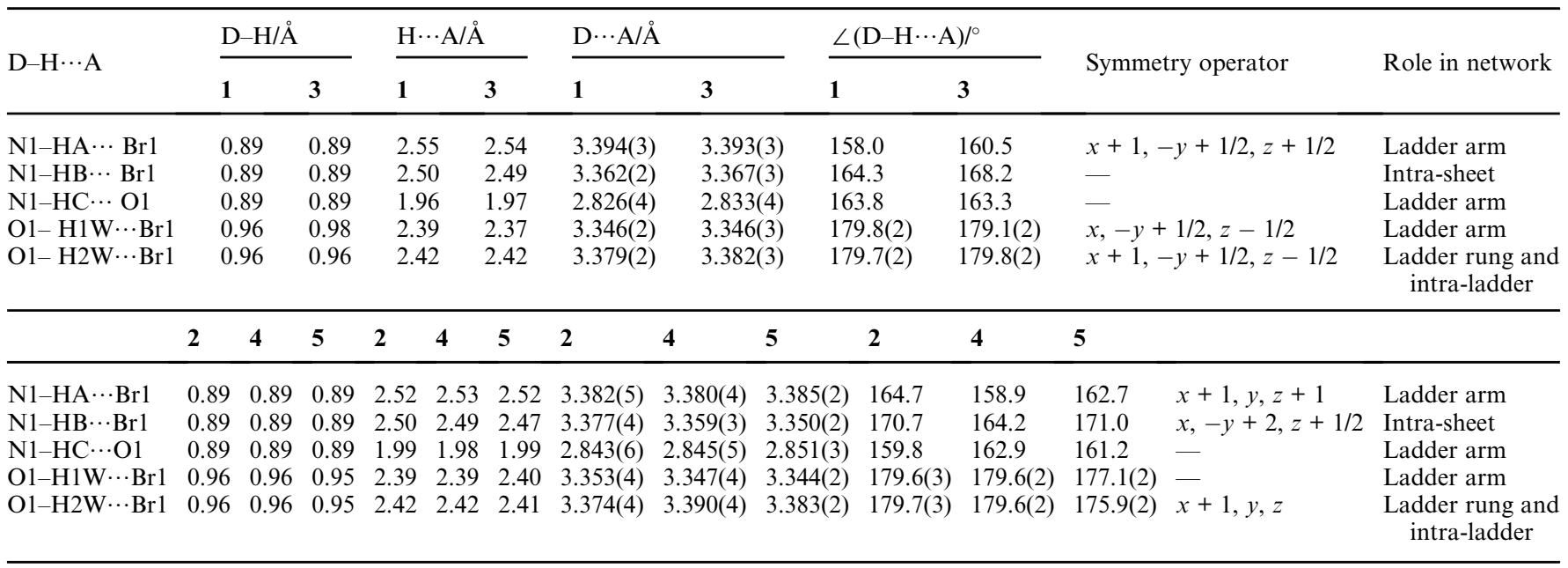

bromide anion in $\mathbf{2}$. In both structures $\mathbf{2}$ and $\mathbf{6}$ the alkyl chains are interdigitated. However, a lower degree of interdigitation is observed for $\mathbf{2}$ where atoms are interdigitated from atom C(3), with $3.82 \AA$ of the cation, measured from the ammonium group, not interdigitated. In structure $\mathbf{6}$ interdigitation already starts at atom $\mathrm{C}(2)$, and only $2.51 \AA$ of the cation is not interdigitated. The result of the larger degree of interdigitation in $\mathbf{6}$ is a higher packing efficiency ${ }^{21}$ compared to $2(64.7 \%$ for 2 and $65.1 \%$ for $\mathbf{6})$.

It has been reported ${ }^{22}$ that water molecules insert into $\mathrm{N}-\mathrm{H} \cdots$ $\mathrm{Cl}-\mathrm{M}$ hydrogen bonds, but not into $\mathrm{C}-\mathrm{X} \cdots \mathrm{Cl}-\mathrm{M}$ hydrogen bonds, where $\mathrm{X}$ is a halogen. The present study highlights the ability of water molecules to insert into $\mathrm{N}^{+}-\mathrm{H} \cdots \mathrm{Br}^{-}$hydrogen bonds. The hydrogen bonding network in $\mathbf{6}$ can also be described by ladder stacking, ${ }^{10}$ and the resultant hydrogen bonding network is a single corrugated, two-dimensional sheet consisting of individual ladders, as illustrated schematically in Fig 5a. Fig. 5b illustrates the positions where water molecules are inserted to obtain one two-dimensional sheet observed in $\mathbf{2}$, with the hydrogen bonding network obtained after insertion of water molecules illustrated in Fig. 5c. Water molecules insert into half 


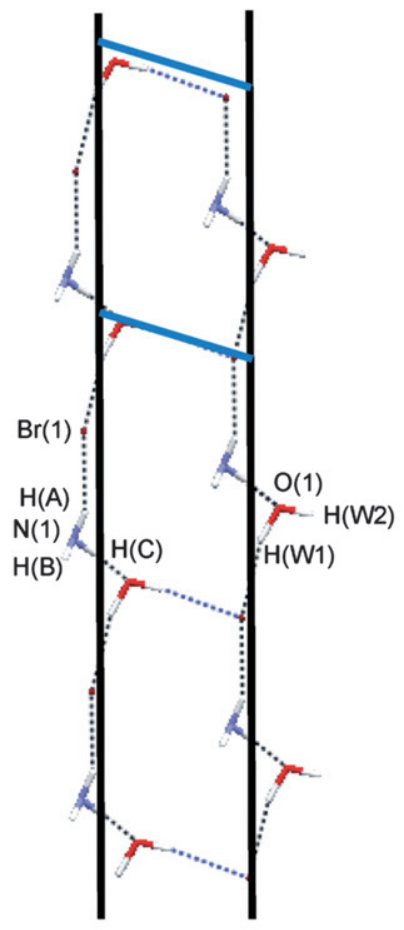

(a)

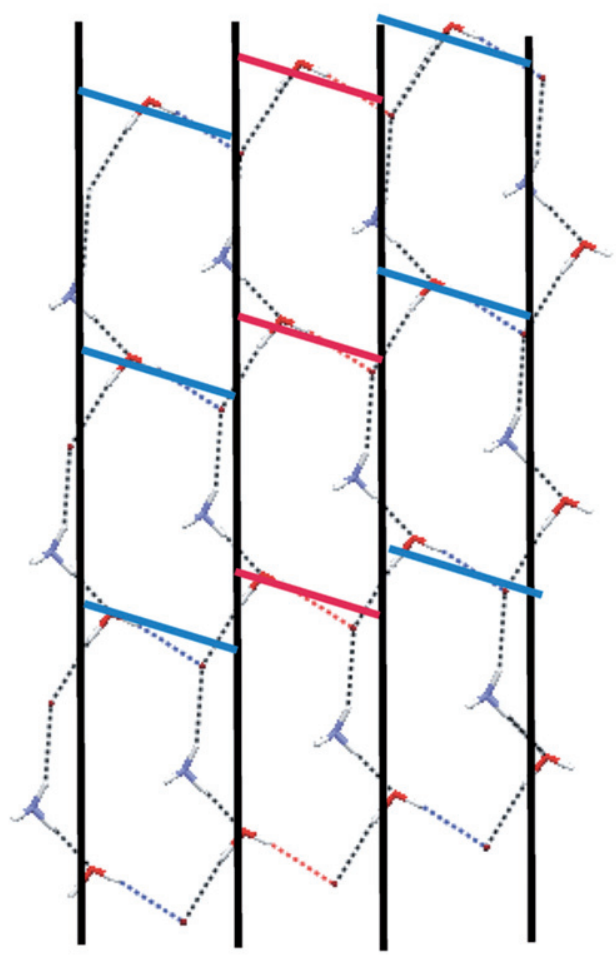

(b)

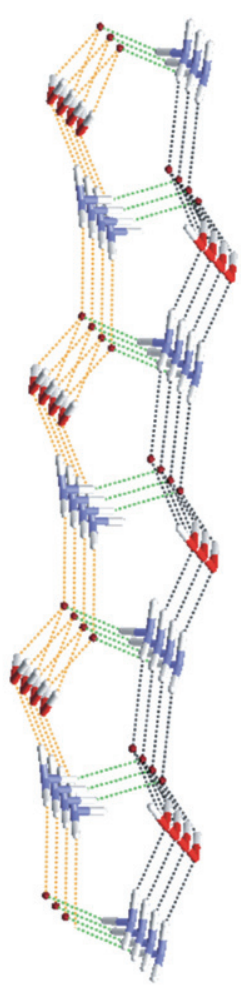

(c)

Fig. 3 (a) Hydrogen bonded ladder with ladder arms in black and rungs in blue. (b) Red intra-ladder interactions connecting ladders to form 2-d sheets. (c) Green intra-sheet interactions connect orange and black sheets to form a hydrophilic bilayer.

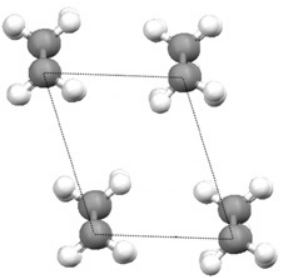

(a)

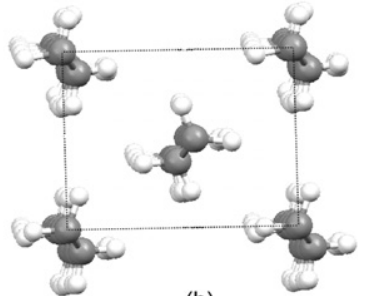

(b)
Fig. 4 (a) $\mathrm{M}_{2}^{\|}$methylene subcell exhibited by structures 1 to 5 (b) $\mathrm{O} \perp^{\prime}$ methylene subcell exhibited by structure 6 .

of the $\mathrm{N}^{+}-\mathrm{H} \cdots \mathrm{Br}^{-}$interactions occuring in the anhydrate structure to form the two-dimensional network observed in the hydrate. Doubling of this hydrogen bonded sheet gives the hydrophilic bilayer present in structures 1-5.

\section{Thermal analysis}

All the hydrates under investigation exhibit thermotropic polymorphism corresponding to dehydration and stepwise melting. It has been reported for the $n$-alkylammonium chloride salts that the higher-temperature phase transitions give rise to conformational defects in the hydrophobic layers, and/or rearrangements in the hydrophilic layers. ${ }^{15}$ Phase transition temperatures observed for compounds $\mathbf{1}$ to $\mathbf{5}$ are listed in Table 4, and DSC scans illustrated in Fig. 6.

Roman numerals are employed to distinguish between different high temperature phases. The even carbon chain

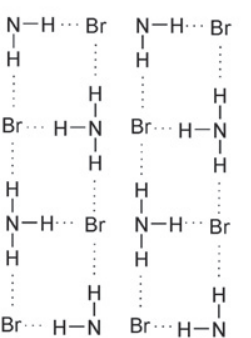

(a)

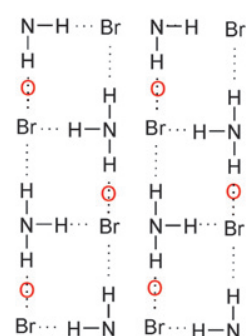

(b)

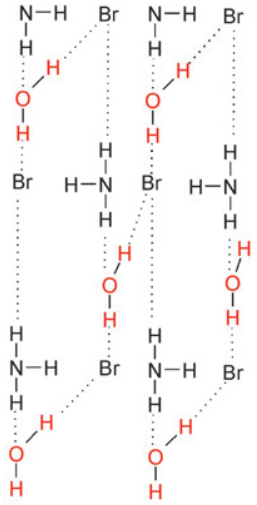

(c)
Fig. 5 (a) Schematic representation of hydrogen bonding network in 6 (b) Positions of water molecule insertion in network (c) Two-dimensional network formed in $\mathbf{2}$.

compounds exhibit three low-temperature, endothermic phase transitions on heating, while the odd carbon chain compounds show only two. It is proposed that this difference is the result of the formation of a different dehydration product for the oddand even carbon compounds. The first endotherm observed in the DSC scans corresponds to the irreversible dehydration of compounds 1 to 5. Dehydration occurs at a higher temperature for the odd carbon chain compounds $\left(T(\mathrm{~K})_{\text {dehydration, odd }}=3.34 n\right.$ +285.51 , where $n$ is the number of carbon atoms) compared to the even chain compounds $\left(T(\mathrm{~K})_{\text {dehydration,even }}=3.78 n+277.08\right)$, 
Table 4 Phase transition temperatures and enthalpies for compounds $\mathbf{1}$ to

\begin{tabular}{|c|c|c|c|c|c|c|c|c|c|c|}
\hline \multirow[b]{2}{*}{ Transition } & \multicolumn{2}{|l|}{1} & \multicolumn{2}{|l|}{2} & \multicolumn{2}{|l|}{3} & \multicolumn{2}{|l|}{4} & \multicolumn{2}{|l|}{5} \\
\hline & Onset & Peak & Onset & Peak & Onset & Peak & Onset & Peak & Onset & Peak \\
\hline hydrate $\rightarrow \beta$ (odd) & 328.23 & 329.56 & - & - & 336.65 & 337.25 & - & - & - & - \\
\hline hydrate $\rightarrow \phi$ (even) & - & - & 330.00 & 333.55 & - & - & 337.56 & 340.39 & 345.13 & 348.33 \\
\hline$\phi \rightarrow \beta$ (even) & - & - & 345.62 & 347.01 & - & - & 354.06 & 356.12 & 359.38 & 360.6 \\
\hline liquid crystal $\rightarrow$ melt & 515.77 & 517.82 & 510.31 & 510.99 & 500.53 & 501.38 & 492.91 & 493.82 & 476.98 & 477.96 \\
\hline
\end{tabular}

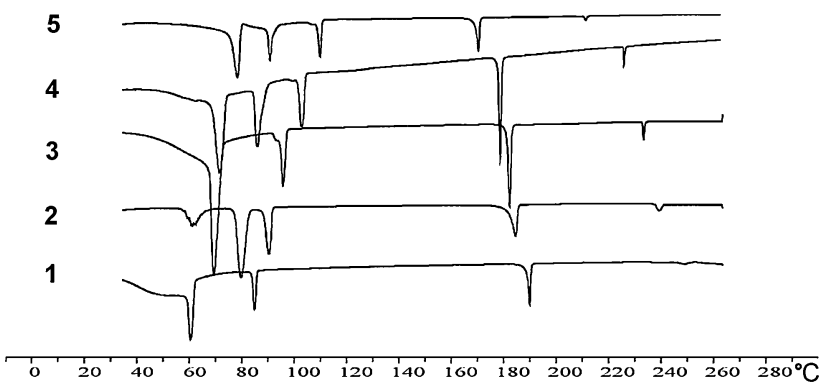

Fig. 6 DSC scans for compounds 1 to 5 .

and overall there is a trend of increasing dehydration temperature with increasing alkyl chain length. The dehydration was confirmed by TGA, with the observed weight loss corresponding to the removal of all water molecules from the lattice.

An intermediate phase, (i), is obtained after dehydration of the even carbon members, a phase that does not exist for the odd carbon chain compounds. After a second thermotropic transition the even carbon chain compounds are in the same phase, phase (ii), as the odd chain compounds after dehydration. On further heating two more phase transitions, from phase (ii) to phase (iii), and phase (iii) to a liquid crystal phase, occur. A low-enthalpy transition to the melted phase follows. The last two phase transitions show a decrease in transition temperature with an increase in carbon chain length. Although apparently anomalous, this effect may be rationalised by assuming an increase in dynamical disorder associated with conformational changes as the alkyl chain gets longer, thus requiring less energy on phase transition, resulting in lower transition temperatures. Transition temperatures from phase (ii) to phase (iii) are given by the equation $T(\mathrm{~K})$ $=5.01 n+288.98$, while transition temperatures of phase (iii) to the liquid crystal phase is given by $T(\mathrm{~K})=-3.91 n+507.25$, and the final melting temperature by $T(\mathrm{~K})=-7.92 n+619.77$.

\section{Conclusions}

All the compounds investigated self-assemble to form layered structures consisting of interdigitated hydrocarbon layers and ionic bilayers. These structures are the result of a delicate balance between the van der Waals interactions involving the hydrocarbon chains and the strong, charge assisted hydrogen bonds that occur in the hydrophilic layer. Deviations from the ideal alltrans long-chain geometry occur in the vicinity of the ammonium group due to the flexible nature of the long-chain allowing the hydrogen bonding ammonium group to be orientated in a way that maximises hydrogen bond strength while still maintaining favourable hydrophobic interactions through parallel alkyl chains.

The incorporation of water molecules into half of the $\mathrm{N}^{+}-\mathrm{H} \cdots$ $\mathrm{Br}^{-}$hydrogen bonds occurs, and this induces a number of important structural changes in the hydrates compared to the anhydrous structure of $n$-dodecylammonium bromide. ${ }^{3}$ Tilted chains are observed in the anhydrous structure but approximately perpendicular chains are present in the hydrates. The hydrates also exhibit a different methylene subcell packing and a reduced degree of interdigitation, resulting in a lower packing efficiency, compared to the anhydrous structure.

Thermotropic phase transitions occur in all the compounds, with the odd carbon chain compounds undergoing four transitions to reach the melted phase, and the even carbon chain compounds five, indicating their potential use as solid-solid phase change materials.

\section{Experimental}

\section{Crystallization of materials}

Compounds 1 to 5 were synthesised by dissolving $1.00 \mathrm{~g}$ of the solid $n$-alkylamine in $40 \mathrm{ml}$ of chloroform (Aldrich) ( $n$-tridecylamine, 98\%, Aldrich; $n$-tetradecylamine, 95\%, Aldrich; $n$-pentadecylamine, 96\%, Aldrich; $n$-hexadecylamine, 98\%, Aldrich; $n$-octadecylamine, 97\%, Aldrich). Dissolution was aided by slight heating of the solution on a hotplate. A 1.5 stoichiometric excess of hydrobromic acid (48\%, Aldrich) was added dropwise to the hot amine solution. Crystallization of the salt was encouraged by placing the warm solution on ice. Crystals of 1, 3, 4 and 5 were obtained directly from cooling the chloroform solution on ice. Crystals of $\mathbf{2}$ were obtained by slow crystallization from a chloroform solution, at room temperature, open to the air.

\section{Single crystal X-ray diffraction}

Intensity data were collected on a Bruker SMART 1K CCD area detector diffractometer with graphite monochromated Mo $\mathrm{K} \alpha$ radiation $(50 \mathrm{kV}, 30 \mathrm{~mA})$. Data for compounds $\mathbf{1 - 5}$ were collected at $20^{\circ} \mathrm{C}$. The collection method involved $\omega$-scans of width $0.3^{\circ}$. Data reduction was performed using the program SAINT $+{ }^{23}$ (Version 6.02), and absorption corrections were made using the program SADABS. ${ }^{24}$ The crystal structures were solved by direct methods using SHELXS-9725 as part of the WinGX ${ }^{26}$ 
suite. In structures $\mathbf{1}, \mathbf{2}$ and $\mathbf{3}$ hydrogen atoms on the water molecule were located in the difference map, fixed at this position, and not refined further, and in structures $\mathbf{4}$ and $\mathbf{5}$ hydrogen atoms on the water molecule were placed in calculated positions, approximately $0.96 \AA$ along the vector between the $\mathrm{O}$ donor and $\mathrm{Br}$ acceptor atoms. All other hydrogen atoms were placed geometrically and allowed to ride on their parent atoms. Diagrams and publication material were generated using WinGX, ${ }^{26}$ ORTEP,${ }^{27}$ PLATON $^{21}$ and Mercury. ${ }^{28}$

\section{DSC and TGA}

DSC samples were analysed on a Mettler Toledo Star ${ }^{\mathrm{e}}$ system. Aluminium sample pans were used, with punctured pan lids. Samples were heated from 273.5 to $538.5 \mathrm{~K}$ at a rate of $5 \mathrm{~K}$ $\mathrm{min}^{-1}$. TGA curves were collected on a Perkin Elmer Pyris 1 instrument. Samples were heated in a platinum pan from 273.5 to $538.5 \mathrm{~K}$ at a constant rate of $5 \mathrm{~K} \mathrm{~min}^{-1}$.

\section{References}

1 See, among others: J. Fàbry, J. Kroupa and I. Císaŕová, Acta Crystallogr., Sect. C: Cryst. Struct. Commun., 2001, 57, 22-25; R. Rudert, B. Schultz, G. Reck, D. Vollhardt and J. Kriwanek, Acta Crystallogr., Sect. B: Struct. Sci., 2000, 56, 124-131; G. Gbabode, P. Negrier, D. Mondieig, E. M. Calvo, T. Calvet and M. A. Cuevas-Diarte, Chem.-Eur. J., 2008, 13, 3150-3159; M. M. Saw, P. Kurz, N. Agorastos, T. S. A. Hor, F. X. Sundram, Y. K. Yan and R. Alberto, Inorg. Chim. Acta, 2006, 359, 4087-4094.

2 See, among others: K. Uno, Y. Ogawa and N. Nakamura, Cryst. Growth Des., 2008, 8, 592-599; K. Wedeking, Z. Mu, G. Kehr, J. C. Sierra, C. M. Lichtenfeld, S. Grimme, G. Erker, R. Fröhlich, L. Chi, W. Wang, D. Zhong and H. Fuchs, Chem.-Eur. J., 2006, 12, 1618-1628; H. Kobayashi, T. Yamamoto and N. Nakamura, Cryst. Res. Technol., 1995, 30, 375-380.

3 See, among others: X. Guo, B. A. Pethica, J. S. Huang and R. K. Prud'homme, Macromolecules, 2004, 37, 5638-5645; A. Holz, J. Naghizadeh and D. T. Vigren, Phys. Rev. B: Condens. Matter Mater. Phys., 1983, 27, 512-522; C. A. Ericsson, L. C. Ericsson, V. Kocherbitov, O. Söderman and S. Ulvenlund, Phys. Chem. Chem. Phys., 2005, 7, 2970-2977; Y. Ogawa and N. Nakamura, Bull. Chem. Soc. Jpn., 1999, 72, 943-943.

4 See, among others: E. Badea, G. D. Gatta, D. D'Angelo, B. Brunetti and Z. Rečková, J. Chem. Thermodyn., 2000, 38, 1546-1552; E. Badea, I. Blanco and G. D. Gatta, J. Chem. Thermodyn., 2007, 39, 1392-1398; N. A. S. White and H. A. Ellis, J. Mol. Struct, 2008, 888, 386-393.

5 R. Boese, H. C. Weiss and D. Bläser, Angew. Chem., Int. Ed., 1999, 38, 988-992.

6 V. R. Thalladi, R. Boese and H. C. Weiss, Angew. Chem., Int. Ed., 2000, 39, 918-922.
7 V. R. Thalladi, R. Boese and H. C. Weiss, J. Am. Chem. Soc., 2000, 122, 1186-1190.

8 A. D. Bond, New J. Chem., 2004, 28, 104-114.

9 V. R. Thalladi, M. Nüsse and R. Boese, J. Am. Chem. Soc., 2000, 112, 9227-9236.

10 K. Schenk and G. Chapuis, Acta Crystallogr., Sect. C: Cryst. Struct. Commun., 1986, 42, 1076-1078.

11 J. Silver and P. J. Marsh, Acta Crystallogr., Sect. C: Cryst. Struct. Commun., 1995, 51, 2432-2434; A. V. A. Pinto, I. Vencato, H. A. Gallardo and Y. P. Mascarenhas, Mol. Cryst. Liq. Cryst., 1987, 149, 29-40; M. Gordon, E. Stenhagen and V. Vand, Acta Crystallogr., 1953, 6, 739-741.

12 B. M. Lundén, Acta Crystallogr., Sect. B: Struct. Crystallogr. Cryst. Chem., 1974, 30, 1756-1760.

13 J. Silver, S. Martin, P. J. Marsh and C. S. Frampton, Acta Crystallogr., Sect. C: Cryst. Struct. Commun., 1996, 52, 1261-1263.

14 G. J. Kruger, M. Rademeyer and D. G. Billing, Acta Crystallogr., Sect. E: Struct. Rep. Online, 2003, 59, o480-o482.

15 See, among others: K. J. Schenk, G. Chapuis, R. Blinc and J. Seliger, J. Mol. Struct., 1988, 176, 331-335; E. C. Reynhardt, Chem. Phys. Lett., 1996, 256, 548-554; E. C. Reynhardt, Aust. J. Phys., 1998, 51, 557-575; K. J. Schenk, C. A. Ogle, G. Chapuis, R. Cavagnat, A. Jokic and M. Rey-Lafon, J. Phys. Chem., 1989, 93, 5040-5049; A. Terreros, P. A. Galero-Gomez and E. Lopez-Cabarcos, J. Therm. Anal. Calorim., 2000, 61, 341-350; V. Busico, P. Cernicchiaro, P. Corradini and M. Vacatello, J. Phys. Chem., 1983, 87, 1631-1635; K. Horiuchi, H. Takayama, S. Ishimaru and R. Ikeda, Bull. Chem. Soc. Jpn., 2000, 73, 307-314; S. Tanaka, N. Onoda-Yamamuro, S. Ishimaru and R. Ikeda., Bull. Chem. Soc. Jpn., 1997, 70, 2981-2986.

16 J. Tsau and D. F. R. Gilson, J. Phys. Chem., 1988, 72, 4082-4085.

17 L. Infantes, J. Chisholm and S. Motherwell, CrystEngComm, 2003, 5, 480-486.

18 A. I. Kitaigorodskii, in Organic Chemical Crystallography, Consultants Bureau, New York, 1961, ch. 4, pp. 183-192; E. Segermann, Acta Crystallogr., 1965, 19, 789-796; S. Abrahamsson, B. Dahlen, H. Lofgren and I. Pascher, Progr. Chem. Fats Other Lipids, 1978, 16, 125-143.

19 A. D. Bond, Cryst. Growth Des., 2005, 5, 755-771.

20 M. C. Etter and J. C. MacDonald, Acta Crystallogr., Sect. B: Struct. Sci., 1990, 49, 256-262; J. Grell, J. Bernstein and G. Tinhofer, Acta Crystallogr., Sect. B: Struct. Sci., 1999, 55, 1030-1043.

21 A. L. Spek, J. Appl. Crystallogr., 2003, 36, 7-13.

22 F. Zordan and L. Brammer, Acta Crystallogr., Sect. B: Struct. Sci., 2004, 60, 512-519.

23 Bruker, $S A I N T+$, version 6.02 (includes XPREP and SADABS), Bruker AXS Inc., Madison, WI, USA, 1999.

24 G. M. Sheldrick, $S A D A B S, 1999$, University of Göttingen, Germany.

25 G. M. Sheldrick, SHELX, release 97-2 (includes SHELXS and SHELXL), 1997, University of Göttingen, Germany.

26 L. J. Farrugia, J. Appl. Crystallogr., 1999, 32, 837-838.

27 L. J. Farrugia, J. Appl. Crystallogr., 1997, 30, 565.

28 I. J. Bruno, J. C. Cole, P. R. Edgington, M. K. Kessler, C. F. Macrae, P. McCabe, J. Pearson and R. Taylor, Acta Crystallogr., Sect. B: Struct. Sci., 2002, 58, 389-397. 